\title{
ANALYTIC SOLUTION OF THE STEFAN PROBLEM IN FINITE MEDIUMS
}

\author{
BY
}

\section{A. KAR AND J. MAZUMDER}

University of Illinois at Urbana-Champaign, Urbana, Illinois

\begin{abstract}
The classical Stefan problem is considered in this paper for finite mediums with Dirichlet boundary conditions. Analytic solutions for the temperature distributions and the location of the moving interface are obtained by using Lie group theory and the superposition principle. The existence of analytically exact solutions is established by proving the convergence of the solution.
\end{abstract}

1. Introduction. The Stefan problem is very interesting because of its wide applications in science and engineering and its nonlinearity, which poses an immense challenge to those who seek analytically exact solutions. In casting industries, the melting and solidification of metals are studied extensively to understand the formation of microstructures. The thawing of soils, the formation of ice, and the cooling of large masses of igneous rock are important in geology. Melting and solidification are also encountered in welding, in crystallization, and in the formation of alloys. Recently, lasers are being increasingly used for cutting, drilling, welding, and developing novel metastable alloys, which involve phase changes and moving boundaries. The application of the Stefan problem to study the formation of metastable alloys during laser-aided materials processing can be found in [1-3]. Tao studied the Stefan problem of a polymorphous material [4] and the formation of binary alloys [5, 6].

Studies on phase change problems were first published by Lamé and Clapeyron in 1831 and then by Stefan in 1889. However, the exact solutions for such phase change problems were discussed by Neumann in his lectures in the 1860s, which were published in 1912. The method of obtaining analytic solutions for phase change problems in semi-infinite mediums can be found in [6,7], and it can be seen that the solutions are obtained only for a certain class of problems in which the governing equations as well as the boundary conditions are invariant under a similarity variable which transforms the partial differential equations for the temperature distribution into ordinary differential equations. Tao solved the Stefan problem with arbitrary initial and boundary conditions [9] as well as with arbitrary heat flux and initial conditions [6]. In all these studies, the material has been considered semi-infinite, which can be a good approximation for many cases in which the material is so long 
that the boundary at one end remains unaffected for all practical purposes. However, the semi-infinite medium approximation does not hold good when the material is not very long, and in this situation, the Stefan problem has to be solved for the finite medium.

This paper presents a technique for obtaining an analytic solution of the Stefan problem for finite mediums. Although the Stefan problem with Dirichlet boundary conditions is solved in this paper, the proposed technique can be applied to the Stefan problem with arbitrary heat flux and initial conditions by expanding the boundary and initial conditions in appropriate power series as has been done in [9] and [6], respectively.

2. Mathematical analysis. The formulation of the Stefan problem with Dirichlet boundary conditions in semi-infinite mediums can be found in [7] and [8]. For finite mediums, the one-dimensional phase change problem can be written as

$$
\begin{gathered}
\alpha_{1}\left(\partial^{2} T_{\mathrm{I}} / \partial x^{2}\right)=\partial T_{\mathrm{I}} / \partial t \quad \text { for } 0<x<s(t), \\
\alpha_{2}\left(\partial^{2} T_{\mathrm{II}} / \partial x^{2}\right)=\partial T_{\mathrm{II}} / \partial t \quad \text { for } s(t)<x<w, \\
T_{\mathrm{I}}(0, t)=T_{0}, \quad T_{\mathrm{II}}(w, t)=T_{i}, \quad T_{\mathrm{II}}(x, 0)=T_{i}, \\
T_{\mathrm{I}}(s(t), t)=T_{\mathrm{II}}(s(t), t)=T_{M}, \\
\left.k_{1} \frac{\partial T_{\mathrm{I}}}{\partial x}\right|_{s(t)}-\left.k_{2} \frac{\partial T_{\mathrm{II}}}{\partial x}\right|_{s(t)}=\rho L \frac{d s}{d t}, \\
s(0)=0,
\end{gathered}
$$

where $x$ is the location of a point in the Cartesian coordinate system, $t$ is the time variable, $\alpha_{1}$ and $\alpha_{2}$ are the thermal diffusivities of the solid and liquid phases respectively, $T_{\mathrm{I}}$ and $T_{\mathrm{II}}$ are the temperatures of the solid and liquid phases respectively, and $k_{1}$ and $k_{2}$ are the thermal conductivities of the solid and liquid phases respectively. $s(t)$ is the location of the freezing front, $w$ is the width of the region of interest, $\rho$ is the density of the solid phase, $L$ is the latent heat of freezing, and $T_{M}$ is the freezing temperature of the liquid phase.

The above governing Eqs. (1a, b), the boundary and the initial conditions (2), and the interface conditions $(3 a, b, c)$ refer to the physical situation of the freezing of a liquid which is initially, that is, at $t=0$, bounded in the region $0 \leq x \leq w$, and at the temperature $T_{i}$, which is higher than the freezing temperature, $T_{M}$, of the material. Suddenly, the plane $x=0$ is cooled to a temperature $T_{0}<T_{M}$ so that the solidification takes place.

Group analysis $[10,11]$ shows that the parabolic Eqs. (1a, b) are invariant under the similarity transformation

$$
\eta_{i}=x /\left(4 \alpha_{i} t\right)^{1 / 2}, \quad i=1,2 .
$$

But the boundary and the initial conditions (2) do not allow such a similarity transform. For this reason, we convert the independent variable $x$ to

$$
y=x /(x-w)
$$


such that the region $0 \leq x \leq w$ transforms into the half-space $0 \leq y \leq \infty$, which makes the boundary and the initial conditions (2) admit the similarity variable (4). However, under the transformation (5), the governing Eqs. (1a, b) will not admit the similarity variable (4). To obtain a set of similarity variables that will be admitted by the governing Eqs. ( $1 \mathrm{a}, \mathrm{b})$, the boundary and the initial conditions (2), and the interface conditions $(3 a, b, c)$, we use the following two group generators from [11],

$$
U_{1}=T_{i} \frac{\partial}{\partial T_{i}} \text { and } U_{2}=x \frac{\partial}{\partial x}+2 t \frac{\partial}{\partial t},
$$

where $i=\mathrm{I}$ and II, and observe that a linear combination of the Lie groups of a differential equation is also a group for the differential equation. Combining $U_{1}$ and $U_{2}$, we obtain a new group generator,

$$
U_{3}=x \frac{\partial}{\partial x}+2 t \frac{\partial}{\partial t}-m T_{i} \frac{\partial}{\partial T_{i}}, \quad m=0,1,2, \ldots, \infty,
$$

which yields $A x / t^{1 / 2}$ and $B_{m} T_{i} t^{m / 2}$ as similarity variables, where $A$ and $B_{m}$ are arbitrary constants, and $i=\mathrm{I}$ and II. Using these two similarity variables, we define the following independent and dependent similarity variables in order to obtain nonsimilar solutions for the Stefan problem considered in this study:

$$
\begin{gathered}
\eta_{1}=y w /\left(4 \alpha_{1} t\right)^{1 / 2}, \\
\eta_{2}=y w /\left(4 \alpha_{2} t\right)^{1 / 2}, \\
T_{\mathrm{I}}(x, t)=T_{1}(y(x), t)=\sum_{m=0}^{\infty} t^{m / 2} F_{m}\left(\eta_{1}\right), \\
T_{\mathrm{II}}(x, t)=T_{2}(y(x), t)=\sum_{m=0}^{\infty} t^{m / 2} G_{m}\left(\eta_{2}\right) .
\end{gathered}
$$

Also, we note that as $x \rightarrow s(t), y \rightarrow P(t)$, where $P(t)=s(t) /(s(t)-w)$ because of the transformation (5). Therefore, we can write

$$
\dot{s}(t)=-w \dot{P}(t) /(1-P(t))^{2},
$$

where $\dot{s}(t)=d s(t) / d t$ and $\dot{P}(t)=d P(t) / d t$. Applying the transformations (5), (6a), (7a), and (5), (6b), (7b), respectively, to the governing Eqs. (1a) and (1b), and equating the coefficients of the same powers of $t$, we obtain

$$
F_{m}^{\prime \prime}+2 \eta_{1} F_{m}^{\prime}-2 m F_{m}=\sum_{n=1}^{4} u_{n 1} \eta_{1}^{n-1} F_{m-n}^{\prime}+\sum_{n=1}^{4} v_{n 1} \eta_{1}^{n} F_{m-n}^{\prime \prime},
$$

and

$$
G_{m}^{\prime \prime}+2 \eta_{2} G_{m}^{\prime}-2 m G_{m}=\sum_{n=1}^{4} u_{n 2} \eta_{2}^{n-1} G_{m-n}^{\prime}+\sum_{n=1}^{4} v_{n 2} \eta_{2}^{n} G_{m-n}^{\prime \prime},
$$

for $m=0,1,2, \ldots, \infty$, where, for $i=1$ and $2, u_{1 i}=2 A_{i}, u_{2 i}=-12 A_{i}^{2}$, $u_{3 i}=24 A_{i}^{3}, u_{4 i}=-16 A_{i}^{4}, v_{1 i}=4 A_{i}, v_{2 i}=-12 A_{i}^{2}, v_{3 i}=16 A_{i}^{3}, v_{4 i}=-8 A_{i}^{4}$, 
$u_{n i}=v_{n i}=0$ for $n>m, A_{i}=\alpha_{i}^{1 / 2} / w$, and the single and double primes denote, respectively, the first and second derivatives with respect to $\eta_{1}$ in Eq. (9) and with respect to $\eta_{2}$ in Eq. (10).

Similarly, in terms of the new variables defined by the expressions (5), $(6 a, b)$, and $(7 a, b)$, the boundary, initial, and interface conditions can be written as follows. Referring to the boundary and initial conditions (2), we find that the boundary condition at $x=0$ becomes

$$
F_{m}(0)=T_{0} \delta_{m 0}, \quad m=0,1,2,3, \ldots, \infty,
$$

and the boundary condition at $x=w$ and the initial condition become

$$
G_{m}(\infty)=T_{i} \delta_{m 0}, \quad m=0,1,2, \ldots, \infty,
$$

where $\delta_{m 0}$ is the Kronecker delta. The interface condition (3a) can be written as

$$
T_{1}(P(t), t)=T_{2}(P(t), t)=T_{M} .
$$

To simplify (12a), we express $P(t)$ in terms of $t^{1 / 2}$ as

$$
P(t)=2 \alpha_{1}^{1 / 2} \sum_{m=0}^{\infty} a_{m} \tau^{m+1}
$$

where $\tau=t^{1 / 2}$ and $a_{0} \neq 0$. Because of (12b), the interface condition (12a) can be written as $\psi_{1}(\tau)=\psi_{2}(\tau)=T_{M}$, where $\psi_{1}(\tau)=T_{1}\left(P, \tau^{2}\right)$ and $\psi_{2}(\tau)=T_{2}\left(P, \tau^{2}\right)$. Expanding $\psi_{1}(\tau)$ and $\psi_{2}(\tau)$ in Maclaurin series, and equating the coefficients of the same powers of $\tau$, we obtain

$$
\left.T_{1}(P, t)\right|_{\tau=0}=\left.T_{2}(P, t)\right|_{\tau=0}=T_{M} \quad \text { and }\left.\quad \frac{d^{n} T_{1}(P, t)}{d \tau^{n}}\right|_{\tau=0}=\left.\frac{d^{n} T_{2}(P, t)}{d \tau^{n}}\right|_{\tau=0}=0
$$

for $n=1,2,3, \ldots, \infty$, which can be rewritten by using the transformations (7a) and $(7 b)$ as

$$
F_{0}\left(\eta_{1 i}\right)=G_{0}\left(\eta_{2 i}\right)=T_{M}
$$

and

$$
\left.\sum_{m=0}^{n} \frac{d^{n}}{d \tau^{n}}\left\{\tau^{m} F_{m}\left(\eta_{1}\right)\right\}\right|_{\substack{\tau=0 \\ \eta_{1}=\eta_{1 i}}}=\left.\sum_{m=0}^{n} \frac{d^{n}}{d \tau^{n}}\left\{\tau^{m} G_{m}\left(\eta_{2}\right)\right\}\right|_{\substack{\tau=0 \\ \eta_{2}=\eta_{2 i}}}=0,
$$

where $n=1,2,3, \ldots, \infty$, and $\eta_{1 i}$ and $\eta_{2 i}$ are, respectively, the values of $\eta_{1}$ and $\eta_{2}$ at the interface, that is, at $y=P(\tau) . \eta_{1 i}$ and $\eta_{2 i}$ can be shown to be equal to $w \sum_{m=0}^{\infty} a_{m} \tau^{m}$ and $w \alpha^{*} \sum_{m=0}^{\infty} a_{m} \tau^{m}$, respectively, where $\alpha^{*}=\left(\alpha_{1} / \alpha_{2}\right)^{1 / 2}$. The interface condition ( $3 b$ ) can be written as

$$
\left.k_{1} \tau \frac{\partial T_{1}}{\partial y}\right|_{y=P(\tau)}-\left.k_{2} \tau \frac{\partial T_{2}}{\partial y}\right|_{y=P(\tau)}=\frac{1}{2} w^{2} \rho L \frac{1}{(1-P)^{4}} \frac{d P}{d \tau} .
$$

Expanding $\tau \frac{\partial T_{1}}{\partial y}, \tau \frac{\partial T_{2}}{\partial y}$, and $\frac{1}{(1-P)^{4}} \frac{d P}{d \tau}$ in Maclaurin series, using the fact that these three terms are equal to $\left(w / 2 \alpha_{1}^{1 / 2}\right) \sum_{m=0}^{\infty} \tau^{m} F_{m}^{\prime}\left(\eta_{1}\right),\left(w / 2 \alpha_{2}^{1 / 2}\right) \sum_{m=0}^{\infty} \tau^{m} G_{m}^{\prime}\left(\eta_{2}\right)$, and 
$\left\{2 \alpha_{1}^{1 / 2} /(1-P)^{4}\right\} \sum_{m=0}^{\infty} a_{m}(m+1) \tau^{m}$ respectively, and equating the coefficients of the same powers of $\tau$, the interface condition (14) can be written as

$$
\begin{aligned}
\left.B_{1} \frac{d^{n}}{d \tau^{n}} \sum_{m=0}^{\infty} \tau^{m} F_{m}^{\prime}\left(\eta_{1}\right)\right|_{\substack{\tau=0 \\
\eta_{1}=\eta_{1 i}}}-\left.B_{2} \frac{d^{n}}{d \tau^{n}} \sum_{m=0}^{\infty} \tau^{m} G_{m}^{\prime}\left(\eta_{2}\right)\right|_{\substack{\tau=0 \\
\eta_{2}=\eta_{2 i}}} \\
=B \frac{d^{n}}{d \tau^{n}}\left\{\frac{1}{(1-P)^{4}} \sum_{m=0}^{\infty} a_{m}(m+1) \tau^{m}\right\}_{\tau=0}
\end{aligned}
$$

for $n=0,1,2,3, \ldots, \infty$, where $B_{1}=w k_{1} / 2 \alpha_{1}^{1 / 2}, B_{2}=w k_{2} / 2 \alpha_{2}^{1 / 2}, B=w^{2} \rho L \alpha_{1}^{1 / 2}$. For $n=0$, the expression (15) becomes

$$
\left.B_{1} F_{0}^{\prime}\left(\eta_{1}\right)\right|_{\eta_{1}=\eta_{1 i}}-\left.B_{2} G_{0}^{\prime}\left(\eta_{2}\right)\right|_{\eta_{2}=\eta_{2 i}}=B a_{0}
$$

and, for $n=1,2,3, \ldots, \infty$, it becomes

$$
\begin{aligned}
\left.B_{1} \sum_{m=0}^{n} \frac{d^{n}}{d \tau^{n}}\left\{\tau^{m} F_{m}^{\prime}\left(\eta_{1}\right)\right\}\right|_{\substack{\tau=0 \\
\eta_{1}=\eta_{1 i}}}-\left.B_{2} \sum_{m=0}^{n} \frac{d^{n}}{d \tau^{n}}\left\{\tau^{m} G_{m}^{\prime}\left(\eta_{2}\right)\right\}\right|_{\substack{\tau=0 \\
\eta_{2}=\eta_{2 i}}} \\
=B \frac{d^{n}}{d \tau^{n}}\left\{\frac{1}{(1-P)^{4}} \sum_{m=0}^{\infty} a_{m}(m+1) \tau^{m}\right\}_{\tau=0} .
\end{aligned}
$$

Also, the condition $(3 \mathrm{c})$ becomes $P(0)=0$, which is already taken into consideration while choosing a polynomial expression for $P(t)$ as given by Eq. (12b). So far, we have expressed the proposed Stefan problem in terms of the similarity variables, and now the solution will be obtained in the next section.

3. Analytic solutions. To solve Eqs. (9) and (10), we note that each of them represents a set of second-order, coupled, linear ordinary differential equations. However, the coupling is such that the inhomogeneous term of the $m$ th differential equation depends on the solutions of the previous differential equations, and therefore, Eqs. (9) and (10) can be solved by successively determining $F_{0}, F_{1}, F_{2}, \ldots$ and $G_{0}$, $G_{1}, G_{2}, \ldots$, respectively. Also, the general solution of the $m$ th differential equation can be obtained by taking a sum of the homogeneous and particular solutions.

For the zeroth-order solution, we set $m=0$ in Eqs. (9) and (10) to obtain $F_{m}^{\prime \prime}+$ $2 \eta_{1} F_{m}^{\prime}=0$ and $G_{m}^{\prime \prime}+2 \eta_{2} G_{m}^{\prime}=0$, which can be solved easily [7, 8], and when the boundary conditions $(11 \mathrm{a}, \mathrm{b})$ and the interface conditions (13a) and (16a) are satisfied, the solutions for $F_{0}$ and $G_{0}$ become

$$
F_{0}\left(\eta_{1}\right)=T_{0}+f_{0} \operatorname{erf}\left(\eta_{1}\right)
$$

and

$$
G_{0}\left(\eta_{2}\right)=T_{i}+g_{0} \operatorname{erfc}\left(\eta_{2}\right),
$$

where $f_{0}=\left(T_{m}-T_{0}\right) / \operatorname{erf}\left(w a_{0}\right), g_{0}=\left(T_{m}-T_{i}\right) / \operatorname{erfc}\left(w \alpha^{*} a_{0}\right)$, and $a_{0}$ is given by the transcendental equation $2 B_{1} f_{0} \exp \left\{-\left(w a_{0}\right)^{2}\right\}+2 B_{2} g_{0} \exp \left\{-\left(w \alpha^{*} a_{0}\right)^{2}\right\}=\pi^{1 / 2} B a_{0}$. It should be noted that the zeroth-order solution is identical to the solution of the Stefan problem in a semi-infinite medium with Dirichlet boundary conditions. 
For higher-order solutions, we set $m=1,2,3, \ldots$ in Eqs. (9) and (10), and the general solutions can be written as

$$
F_{m}\left(\eta_{1}\right)=f_{m} i^{m} \operatorname{erfc}\left(\eta_{1}\right)+\bar{f}_{m} i^{m} \operatorname{erfc}\left(-\eta_{1}\right)+\phi_{1 m}\left(\eta_{1}\right)
$$

where

$$
\begin{aligned}
\phi_{1 m}\left(\eta_{1}\right)= & \sum_{n=0}^{m-1} i^{n} \operatorname{erfc}\left(\eta_{1}\right) \sum_{p=0}^{\infty} f_{m n p} \eta_{1}^{p}+\sum_{n=0}^{m-1} i^{n} \operatorname{erfc}\left(-\eta_{1}\right) \sum_{p=0}^{\infty} \bar{f}_{m n p} \eta_{1}^{p} \\
& +\exp \left(-\eta_{1}^{2}\right) \sum_{p=0}^{\infty} \bar{f}_{m p} \eta_{1}^{p}
\end{aligned}
$$

and

$$
G_{m}\left(\eta_{2}\right)=g_{m} i^{m} \operatorname{erfc}\left(\eta_{2}\right)+\bar{g}_{m} i^{m} \operatorname{erfc}\left(-\eta_{2}\right)+\phi_{2 m}\left(\eta_{2}\right)
$$

where

$$
\begin{gathered}
\phi_{2 m}\left(\eta_{2}\right)=\sum_{n=0}^{m-1} i^{n} \operatorname{erfc}\left(\eta_{2}\right) \sum_{p=0}^{\infty} g_{m n p} \eta_{2}^{p}+\sum_{n=0}^{m-1} i^{n} \operatorname{erfc}\left(-\eta_{2}\right) \sum_{p=0}^{\infty} \bar{g}_{m n p} \eta_{2}^{p} \\
+\exp \left(-\eta_{2}^{2}\right) \sum_{p=0}^{\infty} \overline{\bar{g}}_{m p} \eta_{2}^{p}
\end{gathered}
$$

In each of Eqs. (17a, c), the first two terms on the right-hand side represent the homogeneous solution of the $m$ th equation, which is obtained from [12]. $f_{m}, \bar{f}_{m}$, $g_{m}$, and $\bar{g}_{m}$ are constants which have to be determined by satisfying the boundary and interface conditions. $\phi_{1 m}\left(\eta_{1}\right)$ and $\phi_{2 m}\left(\eta_{2}\right)$ represent the particular solutions of the $m$ th equations, where the constants $f_{m n p}, \bar{f}_{m n p}, \bar{f}_{m p}, g_{m n p}, \bar{g}_{m n p}$, and $\overline{\bar{g}}_{m p}$ can be determined by using the method of undetermined coefficients. The number of terms in the particular solutions $(17 \mathrm{~b}, \mathrm{~d})$ is purposely taken to be infinite, because it increases as the number $m$ of differential equations increases. Since the number of terms in these series will be finite for a given value of $m$, the values of $f_{m n p}$, $\bar{f}_{m n p}, \bar{f}_{m p}, g_{m n p}, \bar{g}_{m n p}$, and $\overline{\bar{g}}_{m p}$ will become zero after a certain value of $p$ for each $m$ when the method of undetermined coefficients is applied. For this reason, infinite series are used in the particular solutions $(17 \mathrm{~b}, \mathrm{~d})$ to maintain the generality of the solutions.

By applying the boundary condition (11a) to Eq. (17a), we obtain

$$
F_{m}\left(\eta_{1}\right)=f_{m}\left\{i^{m} \operatorname{erfc}\left(\eta_{1}\right)-i^{m} \operatorname{erfc}\left(-\eta_{1}\right)\right\}+\phi_{1 m}\left(\eta_{1}\right)-f_{m}^{*} i^{m} \operatorname{erfc}\left(-\eta_{1}\right)
$$

where

$$
f_{m}^{*}=\left\{\bar{f}_{m 0}+\sum_{n=0}^{m-1} f_{m n 0} i^{n} \operatorname{erfc}(0)+\sum_{n=0}^{m-1} \bar{f}_{m n 0} i^{n} \operatorname{erfc}(0)\right\} / i^{m} \operatorname{erfc}(0) .
$$

Similarly, applying the boundary condition (11b) to Eq. (17b), and noting that $i^{m} \operatorname{erfc}(\infty)=0$ for $m \geq 0$ and $i^{m} \operatorname{erfc}(-\infty)=\infty$ for $m \geq 1, \bar{g}_{m}$ and $\bar{g}_{m n}$ can be shown to be zero for $n \geq 0$, and we obtain

$$
G_{m}\left(\eta_{2}\right)=g_{m} i^{m} \operatorname{erfc}\left(\eta_{2}\right)+\phi_{2 m}^{\prime}\left(\eta_{2}\right)
$$


where

$$
\phi_{2 m}^{\prime}\left(\eta_{2}\right)=\sum_{n=0}^{m-1} i^{n} \operatorname{erfc}\left(\eta_{2}\right) \sum_{p=0}^{\infty} g_{m n p} \eta_{2}^{p}+\exp \left(-\eta_{2}^{2}\right) \sum_{p=0}^{\infty} \overline{\bar{g}}_{m p} \eta_{2}^{p} .
$$

To determine $f_{m}, g_{m}$, and $a_{m}$, we will use the interface conditions (13b) and (16b), which can be simplified as follows. The interface condition (13b) can be rewritten as

$$
\sum_{m=0}^{n} A_{n m}=\sum_{m=0}^{n} B_{n m}=0 \quad \text { for } n=1,2, \ldots, \infty,
$$

where

$$
\begin{aligned}
& A_{n m}=\left.\frac{d^{n}}{d \tau^{n}}\left\{\tau^{m} F_{m}\left(\eta_{1}\right)\right\}\right|_{\substack{\tau=0 \\
\eta_{1}=\eta_{1 i}}} \\
&=\left.n ! \sum_{r_{1}=0}^{n-m} \frac{d^{r_{1}} F_{m}\left(\eta_{1}\right)}{d \eta_{1}^{r_{1}}}\right|_{\substack{\tau=0 \\
\eta_{1}=\eta_{1 i}}} w^{r_{1}} \sum_{\gamma_{i}} \frac{a_{1}^{\gamma_{1}} a_{2}^{\gamma_{2}} \cdots a_{n-m}^{\gamma_{n-m}}}{\gamma_{1} ! \gamma_{2} ! \cdots \gamma_{n-m} !}, \\
& \gamma_{1}+\gamma_{2}+\cdots+\gamma_{n-m}=r_{1}, \\
& \gamma_{1}+2 \gamma_{2}+\cdots+(n-m) \gamma_{n-m}=n-m,
\end{aligned}
$$

and

$$
\begin{gathered}
B_{n m}=\left.\frac{d^{n}}{d \tau^{n}}\left\{\tau^{m} G_{m}\left(\eta_{2}\right)\right\}\right|_{\substack{\tau=0 \\
\eta_{2}=\eta_{2 i}}} \\
=\left.n ! \sum_{r_{2}=0}^{n-m} \frac{d^{r_{2}} G_{m}\left(\eta_{2}\right)}{d \eta_{2}^{r_{2}}}\right|_{\substack{\tau=0 \\
\eta_{2}=\eta_{2 i}}}\left(w \alpha^{*}\right)^{r_{2}} \sum_{\beta_{i}} \frac{a_{1}^{\beta_{1}} a_{2}^{\beta_{2}} \cdots a_{n-m}^{\beta_{n-m}}}{\beta_{1} ! \beta_{2} ! \cdots \beta_{n-m} !}, \\
\beta_{1}+\beta_{2}+\cdots+\beta_{n-m}=r_{2},
\end{gathered}
$$

and

$$
\beta_{1}+2 \beta_{2}+\cdots+(n-m) \beta_{n-m}=n-m \text {. }
$$

Here we have used Leibnitz's rule for higher derivatives of products and Faá di Bruno's formula [12] for higher derivatives of functions of a function. The higher derivatives of $F_{m}\left(\eta_{1}\right)$ and $G_{m}\left(\eta_{2}\right)$ can be determined from Eqs. (18) and (19) respectively.

Similarly, by using Leibnitz's rule and Faá di Bruno's formula, the interface condition (15) can be simplified as

$$
B_{1} \sum_{m=0}^{n} C_{n m}-B_{2} \sum_{m=0}^{n} D_{n m}=B \sum_{m=0}^{n} E_{n m} \quad \text { for } n=1,2, \ldots, \infty \text {, }
$$

where

$$
\begin{aligned}
C_{n m} & =\left.\frac{d^{n}}{d \tau^{n}}\left\{\tau^{m} F_{m}^{\prime}\left(\eta_{1}\right)\right\}\right|_{\substack{\tau=0 \\
\eta_{1}=\eta_{1 i}}} \\
& =\left.n ! \sum_{r_{3}=0}^{n-m} \frac{d^{r_{3}} F_{m}^{\prime}\left(\eta_{1}\right)}{d \eta_{1}^{r_{3}}}\right|_{\substack{\tau=0 \\
\eta_{1}=\eta_{1 i}}} w^{r_{3}} \sum_{\delta_{i}} \frac{a_{1}^{\delta_{1}} a_{2}^{\delta_{2}} \cdots a_{n-m}^{\delta_{n-m}}}{\delta_{1} ! \delta_{2} ! \cdots \delta_{n-m} !},
\end{aligned}
$$




$$
\begin{aligned}
& \delta_{1}+\delta_{2}+\cdots+\delta_{n-m}=r_{3}, \\
& \delta_{1}+2 \delta_{2}+\cdots+(n-m) \delta_{n-m}=n-m \text {; } \\
& D_{n m}=\left.\frac{d^{n}}{d \tau^{n}}\left\{\tau^{m} G_{m}^{\prime}\left(\eta_{2}\right)\right\}\right|_{\substack{\tau=0 \\
\eta_{2}=\eta_{2 i}}} \\
& =\left.n ! \sum_{r_{4}=0}^{n-m} \frac{d^{r_{4}} G_{m}^{\prime}\left(\eta_{2}\right)}{d \eta_{2}^{r_{4}}}\right|_{\substack{\tau=0 \\
\eta_{2}=\eta_{2 i}}}\left(w \alpha^{*}\right)^{r_{4}} \sum_{\mu_{i}} \frac{a_{1}^{\mu_{1}} a_{2}^{\mu_{2}} \cdots a_{n-m}^{\mu_{n-m}}}{\mu_{1} ! \mu_{2} ! \cdots \mu_{n-m} !}, \\
& \mu_{1}+\mu_{2}+\cdots+\mu_{n-m}=r_{4}, \\
& \mu_{1}+2 \mu_{2}+\cdots+(n-m) \mu_{n-m}=n-m \text {; }
\end{aligned}
$$

and

$$
\begin{gathered}
E_{n m}=\frac{d^{n}}{d \tau^{n}}\left\{\frac{1}{(1-P)^{4}} \sum_{m=0}^{\infty}(m+1) a_{m} \tau^{m}\right\}_{\tau=0}^{m} \\
=n !(n-m+1) a_{n-m} \sum_{r_{5}=0}^{m} \frac{\left(3+r_{5}\right) !}{3 !}\left(2 \alpha_{1}^{1 / 2}\right)^{r_{5}} \sum_{\rho_{i}} \frac{a_{0}^{\rho_{1}} a_{1}^{\rho_{2}} \cdots a_{m-1}^{\rho_{m}}}{\rho_{1} ! \rho_{2} ! \cdots \rho_{m} !}, \\
\rho_{1}+\rho_{2}+\cdots+\rho_{m}=r_{5}, \\
\rho_{1}+2 \rho_{2}+\cdots+m \rho_{m}=m .
\end{gathered}
$$

The interface conditions (20) and (21) are essentially three conditions which can be used to determine $\left(f_{1}, g_{1}, a_{1}\right),\left(f_{2}, g_{2}, a_{2}\right), \ldots,\left(f_{i}, g_{i}, a_{i}\right), \ldots$ successively. For example, the zeroth-order solutions yield $\left(f_{0}, g_{0}, a_{0}\right)$, which can be used in the interface conditions (20) and (21) to obtain $\left(f_{1}, g_{1}, a_{1}\right)$, and then, using these two sets, the third set $\left(f_{2}, g_{2}, a_{2}\right)$ can be determined, and so on. It should be noted that the coefficients, $A_{n m}, B_{n m}, C_{n m}, D_{n m}, E_{n m}$, are hard to compute because of the nature of the sums involved in the expressions for these coefficients. A technique to evaluate these coefficients will be discussed in the appendix.

4. Existence and convergence. In the last section, we have obtained the solutions (18) and (19) of the proposed Stefan problem, and have given a method for determining the constants $f_{m}, g_{m}$, and $a_{m}$. We shall now establish the existence of these solutions by proving $[13,9]$ convergence of each series that appears in the solutions. Combining (18) with (7a), (19) with (7b), and using the zeroth solutions, we obtain

$$
\begin{aligned}
T_{\mathrm{I}}=F_{0}\left(\eta_{1}\right) & +\sum_{m=1}^{\infty} t^{m / 2} f_{m}\left\{i^{m} \operatorname{erfc}\left(\eta_{1}\right)-i^{m} \operatorname{erfc}\left(-\eta_{1}\right)\right\}-\sum_{m=1}^{\infty} t^{m / 2} f_{m}^{*} i^{m} \operatorname{erfc}\left(-\eta_{1}\right) \\
& +\sum_{m=1}^{\infty} t^{m / 2} \phi_{1 m}\left(\eta_{1}\right) \quad \text { for } \eta_{1}<\eta_{0}
\end{aligned}
$$


and

$$
T_{\mathrm{II}}=G_{0}\left(\eta_{2}\right)+\sum_{m=1}^{\infty} t^{m / 2} g_{m} i^{m} \operatorname{erfc}\left(\eta_{2}\right)+\sum_{m=1}^{\infty} t^{m / 2} \phi_{2 m}^{\prime}\left(\eta_{2}\right) \text { for } \eta_{2}>\eta_{0}
$$

where $\eta_{0}$ is the position of the solid-liquid interface at time $t_{0}$.

The existence of the zeroth-order solutions $F_{0}\left(\eta_{1}\right)$ and $G_{0}\left(\eta_{2}\right)$ is well established. We note that $i^{m} \operatorname{erfc}(-\eta)$ and $i^{m} \operatorname{erfc}(\eta)$ monotonically increase and decrease, respectively, as $\eta$ increases. This implies that

$$
\begin{gathered}
\left|i^{m} \operatorname{erfc}\left(\eta_{1}\right)-i^{m} \operatorname{erfc}\left(-\eta_{1}\right)\right| \leq\left|i^{m} \operatorname{erfc}\left(\eta_{0}\right)-i^{m} \operatorname{erfc}\left(-\eta_{0}\right)\right|, \\
i^{m} \operatorname{erfc}\left(-\eta_{1}\right) \leq i^{m} \operatorname{erfc}\left(-\eta_{0}\right)
\end{gathered}
$$

for $\eta_{1}<\eta_{0}$, and

$$
i^{m} \operatorname{erfc}\left(\eta_{2}\right) \leq i^{m} \operatorname{erfc}\left(\eta_{0}\right) \text { for } \eta_{2}>\eta_{0} \text {. }
$$

Now, for $t<t_{0}$, the convergence of the first and second series in Eq. (22) and the first series in Eq. (23) can be established in the same way as in [9]. The remaining series in Eqs. (22) and (23) involve the particular solutions that represent the effects of the inhomogeneous terms. Since the inhomogeneous terms of the $m$ th differential equations are bounded, and they do not involve any function of the homogeneous solutions of the $m$ th equation, the last series in Eqs. (22) and (23) will converge for $t<t_{0}$. So $T_{\mathrm{I}}$ and $T_{\mathrm{II}}$ are bounded for $t<t_{0}$. Finally, the existence of the interface position $s(t)$ can be established by following the procedure given in [9].

Acknowledgment. A part of this work was made possible by a grant (N00014-89J-1473) from the U.S. Office of Naval Research. Dr. George Yoder is the program manager.

Appendix. Let us evaluate the second sum that appears in the expression for $A_{n m}$ because such a sum is hard to compute. So, we are interested in the following sum:

$$
S_{n m}=\sum_{\gamma_{i}} \frac{a_{1}^{\gamma_{1}} a_{2}^{\gamma_{2}} \cdots a_{N}^{\gamma_{N}}}{\gamma_{1} ! \gamma_{2} ! \cdots \gamma_{N} !}
$$

such that

$$
\gamma_{1}+\gamma_{2}+\cdots+\gamma_{N}=M
$$

and

$$
\gamma_{1}+2 \gamma_{2}+\cdots+N \gamma_{N}=N
$$

where $n-m$ and $r_{1}$ of the original expression of $A_{n m}$ have been replaced by $N$ and $M$, respectively, and $M \leq N$. It should be noted that $M, N$, and $\gamma_{i}$ assume only the integral values $M \geq 0, N \geq 0$, and $\gamma_{i} \geq 0$ for $i=1,2,3, \ldots, N$.

The difficulty in computing $S_{n m}$ (see Eq. (24)) arises due to the fact that Eqs. (25a, b) involve more than two unknowns for $N>2$. For this reason, a trial and error approach has to be taken to determine all possible values of $\gamma_{i}$ for $i=1,2, \ldots, N$ for a given value of $N$. However, we notice from Eqs. $(25 \mathrm{a}, \mathrm{b})$ that

$$
\operatorname{Max}\left\{\gamma_{i}\right\} \leq \operatorname{Min}\left\{M, C_{i}\right\},
$$


where $C_{i}=\operatorname{Int}\{N / i\}$ for $i=1,2,3, \ldots, N$. Here $\operatorname{Int}\{N / i\}$ refers to the integral value of the ratio $N / i$.

For example, when $N=2$, the admissible values of $M$ are 0,1 , or 2 because $0 \leq M \leq N$. We will determine all possible values of $\gamma_{1}$ and $\gamma_{2}$ by taking $M=2$ because this will allow us to select the values of $\gamma_{1}$ and $\gamma_{2}$ for $m=1$ also. We note that $\operatorname{Max}\left\{\gamma_{1}\right\} \leq 2$ and $\operatorname{Max}\left\{\gamma_{2}\right\} \leq 1$. So the possible values of $\gamma_{1}=0,1,2$, and $\gamma_{2}=0,1$. Now, for a particular value of $\gamma_{1}$, we use all the values of $\gamma_{2}$ and check which combination satisfies Eq. (25b) and yields an admissible value for $M$ from Eq. (25a). For the present case, we find that $\gamma_{1}=0$ and $\gamma_{2}=1$, and $\gamma_{1}=2$ and $\gamma_{2}=0$ are two possible solutions for $M=1$ and 2, respectively, which can be verified easily by solving Eqs. $(25 \mathrm{a}, \mathrm{b})$ for $M=1$ and 2 . This procedure can be extended to any value of $N$, and can also be applied to determine the coefficients, $B_{n m}, C_{n m}, D_{n m}, E_{n m}$, because they all involve a sum similar to Eq. (24).

\section{REFERENCES}

[1] A. Kar and J. Mazumder, One-dimensional diffusion model for extended solid solution in laser cladding, J. Appl. Phys. 61, 2645-2655 (1987)

[2] A. Kar and J. Mazumder, One-dimensional finite-medium diffusion model for extended solid solution in laser cladding of $\mathrm{Hf}$ on nickel, Acta Metall. 36, 701-712 (1988)

[3] A. Kar and J. Mazumder, Extended solid solution and nonequilibrium phase diagram for Ni-Al alloy formed during laser cladding, Met. Trans. A 20A, 363-371 (1989)

[4] L. N. Tao, The Stefan problem of a polymorphous material, J. Appl. Mech. 46, 789-794 (1979)

[5] L. N. Tao, On solidification of a binary alloy, Quart. J. Mech. Appl. Math. 33, 211-225 (1980)

[6] L. N. Tao, Solidification of a binary mixture with arbitrary heat flux and initial conditions, Arch. Rat. Mech. Anal. 76, 167-181 (1981)

[7] H. S. Carslaw and J. C. Jaeger, Conduction of Heat in Solids, 2nd ed., Oxford Univ. Press, 1959

[8] M. N. Özisik, Heat Condition, John Wiley and Sons, New York, 1980

[9] L. N. Tao, The Stefan problem with arbitrary initial and boundary conditions, Quart. Appl. Math. 36, 223-233 (1978)

[10] L. V. Ovsiannikov, Group Analysis of Differential Equations, translation edited by W. F. Ames, Academic Press, New York, 1982, pp. 68-73

[11] G. W. Bluman and G. D. Cole, Similarity Methods for Differential Equations, Springer, New York, 1974, p. 211

[12] M. Abramowitz and I. A. Stegun (editors), Handbook of Mathematical Functions with Formulas, Graphs, and Mathematical Tables, National Bureau of Standards, 1972, p. 299, p. 823

[13] D. V. Widder, The Heat Equation, Academic Press, New York, 1975, pp. 169-194 